\title{
Are We Overradiating Patients with Irritable Bowel Syndrome?
}

\author{
Yousaf B. Hadi Adnan Aman Khan Syeda F.Z. Naqvi Salman Khan \\ Jesse Thompson Justin T. Kupec
}

Internal Medicine, West Virginia University, Morgantown, WV, USA

\section{Keywords}

Irritable bowel syndrome · Medical radiation - Cancer .

Diagnostic imaging

\begin{abstract}
Introduction: Protracted exposures to small doses of radiation, even cumulative effective doses (CED) as low as 50-100 $\mathrm{mSv}$, may increase the risk for malignancy. Medical radiation exposure has not been rigorously examined for patients with irritable bowel syndrome (IBS). We examined medical radiation exposure in patients with IBS at a tertiary care center in the USA. Methods: Patients diagnosed with IBS at our institute from 2009 to 2018 were included in a retrospective cohort study. Medical charts were examined to calculate total and annual CED. Results: 221 patients were included; mean CED was $40.32 \mathrm{mSv}$ (SD: 54.36). Fifty-nine participants (26.7\%) received $>50 \mathrm{mSv}$ of CED with 27 participants (12.2\%) exceeding $100 \mathrm{mSv}$. Conventional imaging, nuclear medicine, and fluoroscopy accounted for $74.08,12.93$, and $12.98 \%$ of total CED, respectively. CT scans contributed to $66.61 \%$ of total CED. Outpatient orders accounted for $37.96 \%$ of total CED, while $31.4 \%$ of total CED was ordered in the emergency department. Population-specific high total CED was calcu-
\end{abstract}

karger@karger.com www.karger.com/iid

Karger"

GOPEN ACCESS
(C) 2020 The Author(s)

Published by S. Karger AG, Basel

This is an Open Access article licensed under the Creative Commons Attribution-NonCommercial-4.0 International License (CC BY-NC) (http://www.karger.com/Services/OpenAccessLicense), applicable to the online version of the article only. Usage and distribution for commercial purposes requires written permission. lated as $105.65 \mathrm{mSv}$. Multivariable binomial logistic regression model found that comorbid anxiety, chronic pain medication use, and diarrhea-predominant IBS were independently positively associated with population-specific high CED exposure. No significant temporal trend in peri-diagnostic mean CED was found. Conclusion: Patients with IBS receive high amounts of medical radiation, with 1 in 4 patients reaching at-risk levels of $50 \mathrm{mSv}$ or more. Usage of pain medication at home, comorbid anxiety, and IBS-D are independently linked to an increased risk of high CED.

(c) 2020 The Author(s)
Published by S. Karger AG, Basel

\section{Introduction}

Radiological studies, including CT scans, are important diagnostic tools in gastrointestinal disease. With advances in interventional radiology, medical imaging for therapeutic procedures is also contributing to medical radiation exposure. Protracted exposures to small doses of radiation, even a cumulative dose of $50-100 \mathrm{mSv}$, may increase the risk for malignancy $[1,2]$. Previous data suggest that as much as $1.5-2 \%$ of all malignances in the USA

Yousaf B. Hadi

Internal Medicine, West Virginia University

1 Medical Center Drive

Morgantown, WV 26505 (USA)

yousaf.hadi@gmail.com 
may have contribution from diagnostic medical radiation $[1,3]$. While medical radiation exposure has been studied in other gastrointestinal diseases, especially inflammatory bowel disease, in detail $[4,5]$, it has not been rigorously examined for patients with irritable bowel syndrome (IBS).

A retrospective cohort study was performed to examine the use of diagnostic imaging in our cohort of patients with IBS between the years 2009 and 2018. We aimed to identify the cumulative effective dose (CED) of radiation received by these patients in the study period and deduce the risk factors associated with exposure to high total CED.

\section{Materials and Methods}

\section{Study Population}

All patients who had established with primary care at West Virginia University and attended gastroenterology clinics at West Virginia University from 2009 to 2018 were retrospectively identified utilizing institutional electronic health records. Only patients with the diagnosis of IBS were included in our retrospective cohort study. Before commencement, the study was approved by our institutional review board. Patients were identified by ICD-9 and ICD-10 codes 564.1, 564.5, K58*, and K59.1 and included if they had at least 1 year of follow-up with the gastroenterology section. Patients diagnosed with both Rome III and Rome IV criteria were included, and diagnosis was confirmed by reviewing clinic notes from the gastroenterology clinic. Patients were only included if they were established at West Virginia University for primary care and also followed with the digestive disease clinic at West Virginia University.

Patients with organic gastrointestinal diseases, including inflammatory bowel disease (ulcerative colitis, microscopic colitis, and Crohn's disease), celiac disease, pancreatic diseases, and chronic pancreatitis, and all patients with malignancies were excluded. Demographic variables and clinical information (age, gender, comorbidities, type of IBS, and radiological studies performed including the ordering department) were obtained via retrospective chart review.

\section{Diagnostic Radiation Exposure}

Electronic medical records were utilized to identify all imaging studies performed on the included patients from January 1, 2009, to December 31, 2018. Radiation dose estimates were assigned in millisieverts ( $\mathrm{mSv}$ ) and were calculated as effective doses. Effective dose is the standard value for radiation dose estimates as it accounts for radio sensitivities of the involved organs in radiation fields and allows comparison across imaging modalities.

All radiological imaging studies and nuclear medicine scans were assigned an effective radiation dosage utilizing the average effective radiation dosages of imaging studies that have been previously published [6] (Table 1). We included all diagnostic radiological studies performed on patients and then calculated the CED of radiation for each patient by summation of effective doses of all studies performed during the study period. All studies, abdominal
Table 1. Effective radiation dosages of commonly performed radiological examinations

\begin{tabular}{lrl}
\hline Imaging study & $\begin{array}{l}\text { Effective } \\
\text { dose in } \\
\text { mSv }\end{array}$ & $\begin{array}{l}\text { Equivalent } \\
\text { number of chest } \\
\text { radiograph PA }\end{array}$ \\
\hline Chest radiograph PA & 0.02 & 1 \\
Chest radiograph PA/lateral & 0.1 & 5 \\
Barium enema & 8 & 400 \\
CT head & 2 & 100 \\
CT chest & 7 & 350 \\
CT abdomen & 8 & 400 \\
CT abdomen and pelvis & 14 & 700 \\
CT pelvis & 6 & 300 \\
CT for pulmonary embolism & 15 & 750 \\
CT spine & 6 & 300 \\
Small bowel series & 5 & 250 \\
Mammogram & 0.4 & 20 \\
Coronary angiography (diagnostic) & 7 & 350 \\
Endoscopic retrograde & & \\
$\quad$ cholangiopancreatography & 4 & 200 \\
\hline
\end{tabular}

$\mathrm{PA}$, posteroanterior.

and nonabdominal, were included. The ordering department (outpatient, emergency department/urgent care, and inpatient) was recorded.

\section{Change in Diagnostic Radiation Exposure over Time}

In order to test for any difference in the diagnostic radiation exposure over time, separate data for diagnostic radiation administered to patients were collected for radiation exposure during the peri-diagnosis period ( 1 year before and after diagnosis).

\section{Data Aggregation and Analysis}

Data were collected using RedCap, which is an HIPAA compliant data collection tool [7]. Data analysis was performed using $\mathrm{R}$ statistical software [8]. $p$ values $<0.05$ were taken as significant. Univariate analyses were conducted between different population groups by using $t$ test, Mann-Whitney $\mathrm{U}$ test, and $\chi^{2}$ tests. Patients with missing data were excluded from analyses.

The proportion of population receiving $>50$ and $100 \mathrm{mSv}$ of cumulative effective diagnostic radiation dosage during the study period was calculated. Proportion of total CED attributed to emergency department, outpatient orders, and inpatient exposure was also calculated.

A population-specific high CED was defined as radiation dosage above the 90th percentile of the studied population. A population-specific high annual CED was defined as annual radiation dosage above the 90th percentile of the studied population. A binomial logistic regression model was fitted for population-specific high CED by using gender, age at referral/diagnosis, comorbid anxiety or depression, years of follow-up, and type of IBS as covariates. The cumulative CED received due to abdominal imaging and the CED received due to outpatient imaging, emergency room or urgent care imaging, and inpatient imaging were calculated. 
Table 2. Characteristics of the study population

\begin{tabular}{lc}
\hline Variable & $N(\%)$ \\
\hline Age & 40.68 years (SD: 12.08) \\
Male gender & $50(22.62)$ \\
Smoking history & $77(34.8)$ \\
Comorbid & \\
$\quad$ Anxiety & $81(36.65)$ \\
$\quad$ Depression & $81(36.65)$ \\
Chronic pain medication use & $27(12.22)$ \\
\hline
\end{tabular}

\section{Results}

A total of 221 patients were included in the study. Mean age of participants was 40.68 years (SD: 12.08). Mean duration of follow-up after diagnosis was 3.19 years (SD: 1.9) with a range of 1-10. The cohort comprised a majority of females (77.38\%). Baseline characteristics of the study population are summarized in Table 2 .

Mean cumulative effective radiation dose of the study population was $40.32 \mathrm{mSv}$ (SD: 54.36 ). Eight patients did not have any radiological study performed during the study period (3.62\%). Fifty-nine participants $(26.7 \%)$ received $>50 \mathrm{mSv}$ of CED in the study period, and the CED exceeded $100 \mathrm{mSv}$ in 27 participants (12.2\%).

Conventional imaging studies, nuclear medicine studies, and fluoroscopy accounted for 74.08, 12.93, and $12.98 \%$ of total CED, respectively. Chest X-ray was the most commonly performed imaging test but contributed to $<1 \%$ of total CED. Mammograms and CT scan of the abdomen and pelvis were the 2 nd and 3rd most frequent radiological examinations (364 and 220, respectively). CT scans were associated with the highest contribution to CED (66.61\%), of which abdominal and pelvic CT scans were associated with the highest contribution to CED $(3,536 \mathrm{mSv})$. Details of imaging tests are summarized in Table 3.

Outpatient physician orders accounted for $37.96 \%$ of total CED, while $31.4 \%$ of total CED was ordered in the emergency department. Inpatient studies accounted for $30.86 \%$ of total CED (Table 4 ).

The population-specific high total CED (>90th percentile of the sample) was calculated as $105.65 \mathrm{mSv}$. Univariate analyses revealed that comorbid anxiety ( $p$ value 0.00082 ), depression ( $p$ value 0.0008$)$, hyperlipidemia ( $p$ value 0.003 ), diabetes mellitus ( $p$ value 0.025 ), pain medication at home ( $p$ value 0.0001 ), and diarrhea-predominant IBS ( $p$ value 0.03 ) were significantly associated with high population-specific CED (Table 5). The distribution
Table 3. Total number of the frequently obtained radiological tests $(>0.1 \mathrm{mSv}$ only)

\begin{tabular}{lrrr}
\hline Imaging study & $\begin{array}{l}\text { Before } \\
\text { diagnosis }\end{array}$ & $\begin{array}{l}\text { After } \\
\text { diagnosis }\end{array}$ & Total \\
\hline CT scans & 27 & 29 & 56 \\
CT abdomen & 106 & 114 & 220 \\
CT abdomen and pelvis & 31 & 68 & 99 \\
CT chest & 71 & 116 & 187 \\
CT head & 18 & 53 & 71 \\
CT neck & 11 & 33 & 44 \\
CT spine (cervical/thoracic/lumbar) & 29 & 28 & 57 \\
CT intravenous pyelogram & 6 & 2 & 8 \\
Other CT scans & 290 & 433 & 742 \\
\hline Total & & & \\
\hline
\end{tabular}

Other radiological studies (effective dose $>0.1 \mathrm{mSv}$ )

Fluoro-esophagogram $13 \quad 13$

Myocardial perfusion study

Diagnostic catheterization

$21 \quad 35$

Percutaneous coronary intervention

Gastric emptying study

Fluoroscopy procedures

Nuclear cholescintigraphy

Mammograms

1

1

12

48

44

88

$\begin{array}{rr}13 & 26 \\ 35 & 56 \\ 7 & 9 \\ 2 & 3 \\ 19 & 31 \\ 79 & 127 \\ 22 & 66 \\ 76 & 364\end{array}$

of CED for some categorical variables is summarized in Figure 1.

A multivariable binomial logistic regression model was then fitted for population-specific high total CED (exceeding $105.65 \mathrm{mSv}$ ) using gender, age, smoking history, age at referral/diagnosis, comorbid anxiety, depression, years of follow-up, diabetes, hyperlipidemia, coronary artery disease, and type of IBS as covariates. Comorbid anxiety, chronic pain medication use, and diarrhea-predominant IBS were independently positively associated with population-specific high CED (Table 5).

Population-specific high annual CED (defined as annual CED $>90$ th percentile of the study population) was calculated as $12.23 \mathrm{mSv} /$ year. A multivariable binomial logistic regression model was then fitted for populationspecific high annual CED (exceeding $12.23 \mathrm{mSv} /$ year) using gender, age, smoking history, age at referral/diagnosis, comorbid anxiety, depression, years of follow-up, diabetes, hyperlipidemia, coronary artery disease, and type of IBS as covariates. Comorbid anxiety, hyperlipidemia, home pain medication use, and diarrhea-predominant IBS were significantly positively associated with high annual population-specific CED, while longer duration of postdiagnosis follow-up was negatively associated with population-specific high annual CED.
Hadi/Khan/Naqvi/Khan/Thompson/ Kupec 
Fig. 1. Scatter plot of CED by gender, comorbid anxiety, and chronic pain medication use. CED, cumulative effective dose.

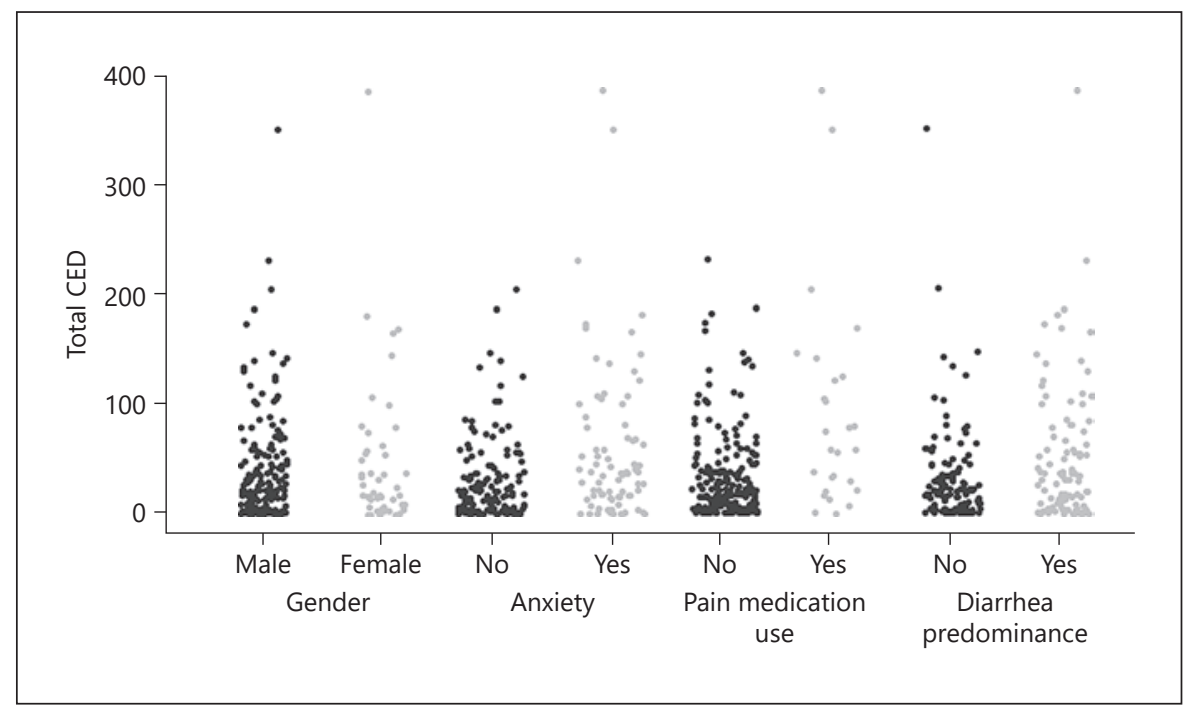

Table 4. Contribution of various types of investigations to total CED of the population

\begin{tabular}{lrl}
\hline Type of radiological study & CED & $\begin{array}{l}\text { Contribution } \\
\text { to total CED, \% }\end{array}$ \\
\hline Emergency department orders & $2,798.83$ & 31.40 \\
Outpatient orders & $3,382.45$ & 37.96 \\
Inpatient orders & $2,750.25$ & 30.86 \\
Nuclear medicine studies & $1,152.6$ & 12.93 \\
Fluoro procedures/studies & 1,157 & 12.98 \\
Conventional radiology & $6,601.92$ & 74.08 \\
\hline
\end{tabular}

CED, cumulative effective dose.

Peri-diagnosis mean effective dose (defined as the effective dose for every patient in the 1 year before and after diagnosis) was calculated for patients diagnosed in each year from 2009 to 2018. Mann-Kendall trend test did not reveal a significant temporal trend in peri-diagnostic mean effective radiation dose over the study period $(p$ value $>0.05$ ).

\section{Discussion}

The prevailing understanding in science regarding cancer risk from radiation is the "linear no threshold" hypothesis, which states that the risk of carcinogenesis from ionizing radiation continues in a linear fashion without a threshold and even a very small radiation dose poses an
Table 5. Univariable and multivariable analyses of the association of different variables with high population-specific total CED

\begin{tabular}{lll}
\hline Variable & $\begin{array}{l}\text { Univariate } \\
\text { test, } \\
p \text { value }\end{array}$ & $\begin{array}{l}\text { Binomial logistic } \\
\text { regression, } \\
p \text { value }\end{array}$ \\
\hline Age (increments of 5) & 0.000 & 0.27 \\
Age at diagnosis (increments of 5) & 0.000 & 0.98 \\
Gender & 0.88 & 0.65 \\
Smoking history & 0.52 & 0.71 \\
Depression & $\mathbf{0 . 0 0 0 8}$ & 0.27 \\
Anxiety & $\mathbf{0 . 0 0 0 8 2}$ & $\mathbf{0 . 0 2}$ \\
Pain medication use & $\mathbf{0 . 0 0 0 1}$ & $\mathbf{0 . 0 0 2}$ \\
Coronary artery disease & 0.08 & 0.47 \\
Diabetes & $\mathbf{0 . 0 2 5}$ & 0.23 \\
Hyperlipidemia & $\mathbf{0 . 0 0 3}$ & 0.11 \\
Follow-up & 0.81 & 0.58 \\
IBS-diarrhea predominant & $\mathbf{0 . 0 3}$ & $\mathbf{0 . 0 0 7}$ \\
\hline
\end{tabular}

Significant associations $(p<0.05)$ are in bold. CED, cumulative effective dose.

increase in cancer risk $[9,10]$. For protracted low-level exposures, scientific data exist regarding increase in cancer risk with doses as low as 50-100 $\mathrm{mSv}$ [1].

Data regarding diagnostic medical radiation exposure in IBS are scarce and heterogeneous. This is the 1st report that has studied medical radiation exposure in IBS patients in the USA. Previously, 2 studies have been reported in the scientific literature from Europe, and although they reported differing results, it was noted that medical radiation exposure in patients with IBS was 
generally at low levels [11]. Desmond et al. [11] studied medical radiation exposure in patients with gastrointestinal disorders and reported minimal radiation exposure in patients with IBS. They reported a mean annual CED of $3.1 \mathrm{mSv}$ for patients with IBS. In their cohort of 629 patients, only 2 patients with functional GI disorders received a total CED $>30.8 \mathrm{mSv}$. In the other study by Englund et al. [12], 5 patients in their sample (3\%) received a total CED of $>50 \mathrm{mSv}$. Our findings are in stark contrast to these previously reported results. We observed a higher annual CED in our population and a very high total $C E D$, with many of these patients being exposed to radiation doses that have been previously associated with higher cancer risk ( $>100 \mathrm{mSv})$. One in 4 patients with IBS in our cohort received a CED of $>50$ $\mathrm{mSv}$. These findings raise significant concern regarding overuse of radiation.

Although medical radiation exposure in IBS patients has not been previously explored in the USA, a study by Master et al. [13] examined the results of CT imaging performed by family practitioners for patients presenting with nonacute abdominal pain and found that it was rarely diagnostic, especially in patients who lacked warning features or red-flag signs. Furthermore, the study commented that incidentally found, unrelated CT findings were prevalent leading to increased healthcare-associated cost. Previous studies have also pointed toward high healthcare resource utilization in patients with IBS that adds unnecessary healthcare-associated cost as well as potentially increasing radiation exposure [14].

These findings are likely related to practice patterns in the USA, having vastly different healthcare practices than in Europe. The European Commission has reported a mean annual CED of medical radiation of $1.1 \mathrm{mSv}$ per year in the European population [15]. In the USA, the National Council on Radiation Protection estimates the mean annual CED to be about $3 \mathrm{mSv} /$ year [16]. Hence, medical radiation exposure may be higher in the USA in general; however, the cumulative exposure we have observed in IBS patients is manifolds higher than that reported in the limited studies from Europe discussed above.

There are clear clinical implications of the findings of our study. We were able to identify risk factors for high radiation exposure. We found that patients with diarrhea-predominant IBS were more likely to receive a high CED. This might be related to confusion with, or concern for, inflammatory bowel disease. A majority of the total CED was attributable to abdominal imaging which supports the hypothesis that these patients were receiv- ing most of their radiation and imaging for gastrointestinal complaints. In patients with an established IBS diagnosis, this is an alarming amount of abdominal radiation. Further, we found a strong significant association of high CED with anxiety and home pain medication use. Previously, it is known that patients with IBS have a high psychiatric comorbidity burden [17]. This finding not only emphasizes the importance of recognizing such a psychiatric comorbidity as a risk factor for high diagnostic medical radiation exposure, but also hints at the possibility that identifying and treating comorbid psychiatric conditions may reduce imaging burden in these patients.

An interesting finding in our study was that the duration of disease or the duration of follow-up was not an independent risk factor for total CED. More than 1 in 10 patients had received $>100 \mathrm{mSv}$ of radiation, and many of them were young patients. Hence, young patients with IBS and newly diagnosed patients should also be considered potentially high risk for a high total CED.

Diagnostic imaging modalities have, no doubt, revolutionized diagnostic medicine, but judicious use of radiological imaging is imperative in reducing the risk that it poses to our patients. Previous studies have shown that physicians are generally unaware of the radiation dosages of imaging modalities. Educational or electronic medical record-based interventions targeted at physicians may be of benefit. We found that the majority of radiation exposure is contributed by outpatient and emergency department encounter providers, which is thus a cohort that should likely benefit from such interventions. The effect of different treatments in patients with IBS on radiation exposure was not studied; however, patients with uncontrolled symptoms would theoretically receive more extraneous imaging and thus effective control of symptoms may lead to reduction in radiation exposure.

There are, however, some inherent limitations in our study. It is likely that the total CED may be underestimated for some patients. Although we included patients who were following in our health system both for primary care and specialist needs, our health system and medical records capture most of our catchment area quite effectively; however, any possible radiological studies conducted at sites uncaptured are unaccounted for. This underestimation, in a way, makes our findings of high radiation exposure even more astonishing. Secondly, like in other reports of medical radiation exposure, we have relied on published standard radiation dosages for the imaging modalities. The amount of radiation exposure actually delivered to each organ varies by patient weight,
36

Inflamm Intest Dis 2021;6:32-37 DOI: $10.1159 / 000511105$
Hadi/Khan/Naqvi/Khan/Thompson/ Kupec 
size, and view adequacy, and thus the standard values may over- or underestimate the radiation exposure in some cases. Our study is also limited by its single center nature.

In conclusion, we have found a high total CED of medical radiation in patients with IBS, with 1 in 4 patients reaching at-risk levels of $50 \mathrm{mSv}$ or more. Usage of pain medication at home, comorbid anxiety, and IBS-D are independently linked to an increased risk of high CED.

\section{Statement of Ethics}

Ethical approval was obtained by the institutional review board at WVU (approval number: 1904526469). Waiver of informed consent was submitted due to the retrospective nature of the study.

\section{Conflict of Interest Statement}

The authors have no conflicts of interest to disclose.

\section{Funding Sources}

No funding was utilized for this study.

\section{Author Contributions}

Yousaf B. Hadi, MD: study design, data analysis, and manuscript writing. Adnan Aman Khan, MD, Syeda F.Z. Naqvi, MD, and Salman Khan, MD: data collection and manuscript writing. Jesse Thompson: data analysis and manuscript writing. Justin $\mathrm{Ku}-$ pec, MD: study design, project supervision, and manuscript writing.

\section{References}

1 Brenner DJ, Doll R, Goodhead DT, Hall EJ, Land CE, Little JB, et al. Cancer risks attributable to low doses of ionizing radiation: assessing what we really know. Proc Natl Acad Sci U S A. 2003;100(24):13761-6.

2 Zakeri N, Pollok RC. Diagnostic imaging and radiation exposure in inflammatory bowel disease. World J Gastroenterol. 2016;22(7): 2165.

3 de Gonzalez AB, Darby S. Risk of cancer from diagnostic X-rays: estimates for the UK and 14 other countries. Lancet. 2004;363(9406): $345-51$.

4 Newnham E, Hawkes E, Surender A, James SL, Gearry R, Gibson PR. Quantifying exposure to diagnostic medical radiation in patients with inflammatory bowel disease: are we contributing to malignancy? Aliment Pharmacol Ther. 2007;26(7):1019-24.

5 Grand DJ, Harris A, Shapiro J, Wu E, Giacalone J, Sands BE, et al. Risk factors for radiation exposure in newly diagnosed IBD patients. Abdom Radiol. 2016;41(7):1363-9.

6 Mettler FA Jr, Huda W, Yoshizumi TT, Mahesh M. Effective doses in radiology and diagnostic nuclear medicine: a catalog. Radiology. 2008;248(1):254-63.
7 Harris PA, Taylor R, Thielke R, Payne J, Gonzalez N, Conde JG. Research electronic data capture (REDCap): a metadata-driven methodology and workflow process for providing translational research informatics support. J Biomed Inform. 2009;42(2):377-81.

8 Team RCR. A language and environment for statistical computing; 2013.

9 Cardis E, Vrijheid M, Blettner M, Gilbert E, Hakama M, Hill C, et al. The 15-country collaborative study of cancer risk among radiation workers in the nuclear industry: estimates of radiation-related cancer risks. Radiat Res. 2007;167(4):396-416.

10 Little MP. Cancer and non-cancer effects in Japanese atomic bomb survivors. J Radiol Prot. 2009;29(2A):A43.

11 Desmond AN, McWilliams S, Maher MM, Shanahan F, Quigley EM. Radiation exposure from diagnostic imaging among patients with gastrointestinal disorders. Clin Gastroenterol Hepatol. 2012;10(3):259-65.
12 Englund H, Lidén K, Lind T, Sundström T, Karling P. Radiation exposure in patients with inflammatory bowel disease and irritable bowel syndrome in the years 2001-2011. Scand J Gastroenterol. 2017;52(3):300-5.

13 Master SS, Longstreth GF, Liu AL. Results of computed tomography in family practitioners' patients with non-acute abdominal pain. Fam Pract. 2005;22(5):474-7.

14 Longstreth GF, Wilson A, Knight K, Wong J, Chiou C-F, Barghout V, et al. Irritable bowel syndrome, health care use, and costs: a US managed care perspective. Am J Gastroenterol. 2003;98(3):600-7.

15 European Commission Medical radiation exposure of the European population. 2014 [cited 2019 Oct 18]. Available from: https:// ec.europa.eu/energy/sites/ener/files/documents/RP180.pdf.

16 Schauer D. Ionizing radiation exposure of the population of the United States. The National Council on Radiation Protection and Measurements (NCRP), Report; 2009.

17 Hausteiner-Wiehle C, Henningsen P. Irritable bowel syndrome: relations with functional, mental, and somatoform disorders. World J Gastroenterol. 2014;20(20):6024. 